\title{
Atom-at-a-time laser resonance ionization spectroscopy of nobelium
}

Mustapha Laatiaoui ${ }^{1,2}$, Werner Lauth ${ }^{3}$, Hartmut Backe ${ }^{3}$, Michael Block ${ }^{1,2,4}$, Dieter Ackermann ${ }^{2} \dagger$, Bradley Cheal $^{5}$, Premaditya Chhetri ${ }^{6}$, Christoph Emanuel Düllmann ${ }^{1,2,4}$, Piet Van Duppen ${ }^{7}$, Julia Even ${ }^{1} \dagger$, Rafael Ferrer ${ }^{7}$, Francesca Giacoppo ${ }^{1,2}$, Stefan Götz ${ }^{1,2,4}$, Fritz Peter Heßberger ${ }^{1,2}$, Mark Huyse ${ }^{7}$, Oliver Kaleja ${ }^{2,8}$, Jadambaa Khuyagbaatar ${ }^{1,2}$, Peter Kunz ${ }^{9}$, Felix Lautenschläger ${ }^{6}$, Andrew Kishor Mistry ${ }^{1,2}$, Sebastian Raeder ${ }^{1,2,7}$, Enrique Minaya Ramirez ${ }^{1} \dagger$, Thomas Walther ${ }^{6}$, Calvin Wraith ${ }^{5}$ \& Alexander Yakushev ${ }^{1,2}$

\begin{abstract}
Optical spectroscopy of a primordial isotope has traditionally formed the basis for understanding the atomic structure of an element. Such studies have been conducted for most elements ${ }^{1}$ and theoretical modelling can be performed to high precision ${ }^{2,3}$, taking into account relativistic effects that scale approximately as the square of the atomic number. However, for the transfermium elements (those with atomic numbers greater than 100), the atomic structure is experimentally unknown. These radioactive elements are produced in nuclear fusion reactions at rates of only a few atoms per second at most and must be studied immediately following their production ${ }^{4}$, which has so far precluded their optical spectroscopy. Here we report laser resonance ionization spectroscopy of nobelium (No; atomic number 102) in single-atom-at-a-time quantities, in which we identify the ground-state transition ${ }^{1} S_{0} \rightarrow{ }^{1} P_{1}$. By combining this result with data from an observed Rydberg series, we obtain an upper limit for the ionization potential of nobelium. These accurate results from direct laser excitations of outer-shell electrons cannot be achieved using state-of-the-art relativistic manybody calculations ${ }^{5-8}$ that include quantum electrodynamic effects, owing to large uncertainties in the modelled transition energies of the complex systems under consideration. Our work opens the door to high-precision measurements of various atomic and nuclear properties of elements heavier than nobelium, and motivates future theoretical work.
\end{abstract}

Since the establishment of the actinide elements in the periodic table, great efforts have been undertaken to investigate their atomic spectra ${ }^{9}$. The prevailing strategy includes deducing many of the atomic properties from detailed knowledge of the electronic configuration, which is subject to strong relativistic effects. This approach has driven optical spectroscopy research programmes for many years, yielding detailed insight into the atomic structure of elements up to einsteinium ${ }^{1}$, including precise values for their ionization potential. The heaviest element for which optical spectroscopy has hitherto been reported is fermium, with atomic number $Z=100$ (ref. 10). A sample of about $10 \mathrm{pg}$ of ${ }^{255} \mathrm{Fm}$ with a half-life of $T_{1 / 2}=20.1 \mathrm{~h}$ facilitated resonance laser ionization spectroscopy experiments ${ }^{11,12}$. In contrast, the transfermium elements do not occur naturally on Earth, and macroscopic samples cannot be synthesized. These elements are typically produced at large accelerator facilities by heavy-ion-induced fusion-evaporation reactions, which yield only low rates. Some of their atomic properties have been accessible in aqueous-phase and gas-phase chemical studies ${ }^{13,14}$. Only recently was the first ionization potential experimentally determined for lawrencium ( $\mathrm{Lr} ; Z=103)$, by applying surface ionization techniques ${ }^{15}$.

Advancing optical studies to be applicable to transfermium elements necessitates fast spectroscopy techniques with extreme sensitivity ${ }^{4}$. Challenges in this research field include producing transfermium atoms, separating them from undesired species that are inevitably co-produced, and manipulating and detecting them within seconds of their production. In our experiments we applied a radiation detected resonance ionization spectroscopy (RADRIS) technique ${ }^{16,17}$, using a two-step photoionization process ${ }^{18}$ for ionizing nobelium atoms $(Z=102)$. Similar techniques have previously been used for laser spectroscopy of short-lived americium fission isomers at production rates as low as ten per second ${ }^{19,20}$. However, an extensive search for atomic transitions within a spectral range proposed by stateof-the-art model calculations is required for such an approach to become feasible for elements such as nobelium. Unambiguous identification of the atoms is achieved via their unique radioactive decay fingerprint ${ }^{21}$.

The isotope ${ }^{254}$ No with a half-life of $T_{1 / 2}=51.2 \mathrm{~s}$ was chosen for the first laser spectroscopy experiments. This isotope was produced in the two-neutron evaporation channel of the complete-fusion reaction ${ }^{48} \mathrm{Ca}+{ }^{208} \mathrm{~Pb}$. The fusion products, emerging from a thin ${ }^{208} \mathrm{~Pb}$ target, were separated in-flight from the intense ${ }^{48} \mathrm{Ca}$ primary beam by the Separator for Heavy Ion reaction Products (SHIP) ${ }^{22}$ at the GSI Helmholtzzentrum für Schwerionenforschung. About four ${ }^{254}$ No ions per second were implanted in a buffer-gas stopping cell installed in the focal plane of the SHIP ${ }^{4}$. These ions were thermalized in 95-mbar high-purity argon gas and accumulated and neutralized on a tantalum catcher filament (see Methods). For a short time during every measurement cycle, the incoming flux of ${ }^{254}$ No ions was turned off and the adsorbed nobelium atoms were evaporated from the filament by heating it briefly to a temperature of about $1,350 \mathrm{~K}$. Nobelium atoms in the ground state $5 f^{14} 7 s^{2}{ }^{1} \mathrm{~S}_{0}$ residing in laser-beam paths in the vicinity of the filament undergo element-selective ionization in a two-step excitation scheme (Fig. 1a inset). The ionization proceeded by resonantly exciting the singlet state $5 f^{14} 7 s 7 p{ }^{1} \mathrm{P}_{1}$ with ultraviolet light from a tunable dye laser (wavelength, $\lambda_{1}$ ), followed by a second excitation into the continuum beyond the ionization potential with ultraviolet light in the wavelength range $349-353 \mathrm{~nm}\left(\lambda_{2}\right)$ from a more powerful excimer laser. Induced photo-ions are subsequently guided by electrostatic fields to a silicon detector where the characteristic $\alpha$ decay of ${ }^{254}$ No is detected ${ }^{23}$.

${ }^{1}$ Helmholtz-Institut Mainz, Staudingerweg 18, D-55128 Mainz, Germany. ${ }^{2}$ GSI Helmholtzzentrum für Schwerionenforschung GmbH, Planckstrasse 1, D-64291 Darmstadt, Germany. ${ }^{3}$ Institut für Kernphysik, Johannes Gutenberg-Universität, Johann-Joachim-Becher Weg 45, D-55128 Mainz, Germany. ${ }^{4}$ Institut für Kernchemie, Johannes Gutenberg-Universität, Fritz-Strassmann Weg 2, D-55128 Mainz, Germany. ${ }^{5}$ Department of Physics, University of Liverpool, Oxford Street, Liverpool L69 7ZE, UK. ${ }^{6}$ Institut für Angewandte Physik, Technische Universität Darmstadt, Schlossgartenstrasse 7, D-64289 Darmstadt, Germany. ${ }^{7}$ KU Leuven, Instituut voor Kern- en Stralingsfysica, Celestijnenlaan 200D, B-3001 Leuven, Belgium. ${ }^{8}$ Institut für Kernphysik, Technische Universität Darmstadt, Schlossgartenstrasse 9, D-64289 Darmstadt, Germany. ${ }^{9}$ TRIUMF, 4004 Wesbrook Mall, Vancouver, British Columbia V6T 2A3, Canada. †Present addresses: Grand Accélérateur National d'lons Lourds, Bd Henri Becquerel, BP 55027 - 14076 Caen Cedex 05, France (D.A.); KVI—Center for Advanced Radiation Technology, Rijksuniversiteit Groningen, Zernikelaan 25, 9747 AA Groningen, The Netherlands (J.E.); Institut de Physique Nucléaire Orsay, 15 rue Georges Clémenceau, 91406 Orsay, France (E.M.R). 


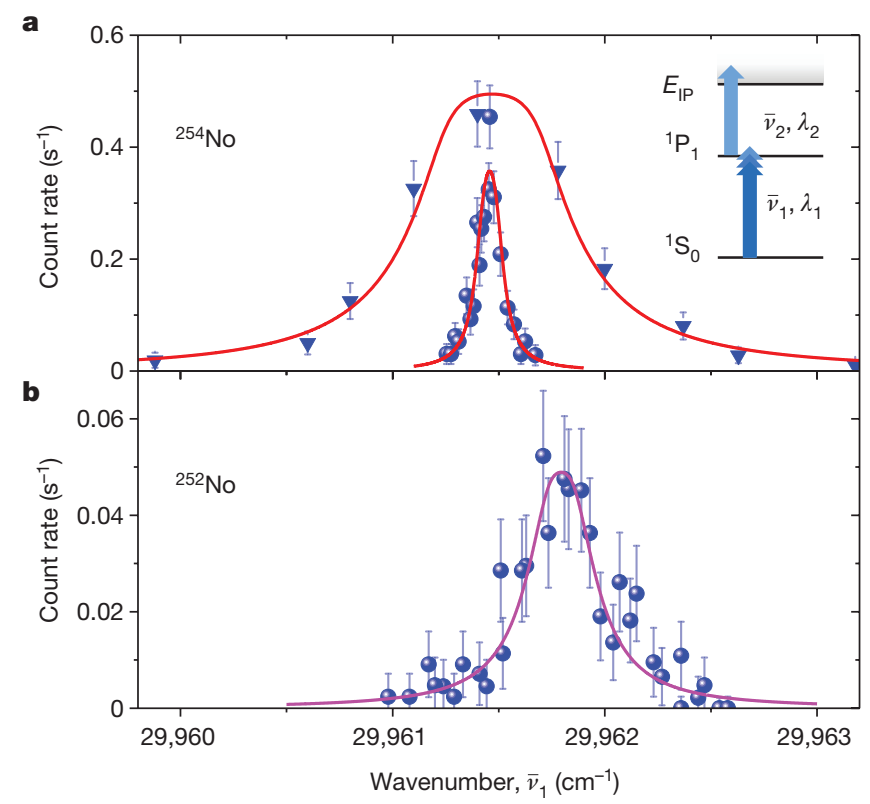

Figure 1 Resonance ionization signals of nobelium atoms. a, Scans over the first-step resonance in ${ }^{254} \mathrm{No}$. The count rates are normalized to a beam current of one particle microampere, which is equivalent to $6.2 \times 10^{12}{ }^{48} \mathrm{Ca}$ projectiles per second. Best fits to the data on the basis of a rate-equation model are indicated by solid lines. Triangles: full-width at half-maximum (FWHM) of the fitted profile, $0.80 \mathrm{~cm}^{-1}$; photon flux, $1.1 \times 10^{14}$ photons per pulse per square centimetre; laser bandwidth, $0.18 \mathrm{~cm}^{-1}$. Spheres: FWHM of the fitted profile, $0.13 \mathrm{~cm}^{-1}$; photon flux, $5.2 \times 10^{12}$ photons per pulse per square centimetre; laser bandwidth, $0.04 \mathrm{~cm}^{-1}$. Inset, a simplified ionization scheme; $E_{\mathrm{IP}}$, ionization potential; $\lambda_{1,2}$, wavelengths of the light used to excite and ionize the atom, $\bar{\nu}_{1,2}=1 / \lambda_{1,2}$, the corresponding wavenumbers. $\mathbf{b}$, Same as a, but for ${ }^{252} \mathrm{No}$. FWHM of the fitted profile, $0.36 \mathrm{~cm}^{-1}$; photon flux, $1.1 \times 10^{13}$ photons per pulse per square centimetre; laser bandwidth, $0.18 \mathrm{~cm}^{-1}$. All error bars indicate \pm 1 s.d.

We chose a spectral range of $28,887-33,191 \mathrm{~cm}^{-1}$ to locate the ${ }^{1} \mathrm{~S}_{0} \rightarrow{ }^{1} \mathrm{P}_{1}$ atomic transition in nobelium (Methods). Latest predictions based on multi-configuration Dirac-Fock (MCDF) and relativistic coupled-cluster calculations ${ }^{5-8}$ suggest wavenumber values between

Table 1 | Comparison of experimental and theoretical values

\begin{tabular}{lll}
\hline Atomic state $5 f^{14} 7 s 7 p^{1} \mathrm{P}_{1}$ in ${ }^{254} \mathrm{No}$ & \\
\hline Method & $\bar{\nu}_{1}\left(\mathrm{~cm}^{-1}\right)$ & $A\left(10^{8} \mathrm{~s}^{-1}\right)$ \\
Experiment & $29,961.457_{-0.007}^{+0.041}$ & $4.2_{-2.8}^{+2.6}$ \\
$\mathrm{Cl}+$ all-order $^{8}$ & $30,203 \pm 600$ & \\
MCDF $^{7}$ & $30,609 \pm 1,100^{*}$ & 3.5 \\
IHFSCC $^{6}$ & $30,056 \pm 800$ & 5.0 \\
MCDF $^{5}$ & $30,650 \pm 800$ & 2.7 \\
\hline lonization potential $^{*}$ & \\
\hline Method & $E_{\mathrm{IP}}\left(\mathrm{cm}^{-1}\right)$ & \\
Experiment $^{-1}$ & \\
Cl + all-order $^{8}$ & $52,467^{\dagger}-53,757.5$ & \\
MCDF $^{7}$ & $54,390 \pm 1,100$ & \\
IHFSCC $^{6}$ & $53,701 \pm 1,100^{\ddagger}$ & \\
Extrapolation $^{29}$ & $53,489 \pm 800^{\ddagger}$ & \\
\hline
\end{tabular}

$\mathrm{Cl}+$ all-order, configuration interaction method combined with the linearized single-double coupled-cluster method (all-order); MCDF, multi-configuration Dirac-Fock; IHFSCC, intermediate Hamiltonian Fock-space coupled cluster; $\bar{\nu}_{1}$, wavenumber of the ${ }^{1} \mathrm{P}_{1}$ state; $A$, Einstein coefficient for spontaneous emission; $E_{\mid \mathrm{P}}$, ionization potential of nobelium.

"Because the error was not explicitly reported in ref. 7, we assessed it from the relative difference between the calculated and measured values for the corresponding state in ytterbium.

TThe lower limit of the ionization potential was estimated from theoretical calculations ${ }^{6}$; see text for details.

‡We give a conservative estimate of the error, the magnitude of which is based on that of the error on the wavenumber of the ${ }^{1} \mathrm{P}_{1}$ state evaluated using the same method.
$29,256 \mathrm{~cm}^{-1}$ and $31,709 \mathrm{~cm}^{-1}$ (Table 1) for the excited state ${ }^{1} \mathrm{P}_{1}$. The rigorous treatment of correlation effects by these approaches improved the predictions given by the first MCDF calculations ${ }^{24}$, which focused on quantum electrodynamic effects. Nonetheless, about 6,110 laserscan steps needed to be conducted before this elusive first-step resonance was discovered. In Fig. 1a (triangles) we show the observed resonance in terms of normalized $\alpha$-decay count rates. A relatively high laser power was initially used, which was favourable for an efficient level search leading to a power-broadened transition. The resonance is centred at a wavenumber of $\bar{\nu}_{1}=1 / \lambda_{1}=29,961.457_{-0.007}^{+0.041} \mathrm{~cm}^{-1}$ (1 s.d. statistical uncertainty) as determined from a subsequent narrow-band scan using an intra-cavity etalon; see Fig. 1a (spheres). The dominant component in the quoted uncertainties originates from the pressure shift (Extended Data Table 1), and results in a relative precision of 1.4 p.p.m., which is much more precise than estimates from modern theories and, hence, provides a powerful benchmark. A comparison of the experimental results for the wavenumber of the ${ }^{1} \mathrm{P}_{1}$ state and theoretical predictions is provided in Table 1.

Having located the resonance in ${ }^{254} \mathrm{No}$, we increased the spectral resolution to measure the isotope dependence of the ${ }^{1} \mathrm{~S}_{0} \rightarrow{ }^{1} \mathrm{P}_{1}$ transition energy by reducing the laser intensity. In Fig. $1 \mathrm{~b}$ we show the measurement of the same transition in ${ }^{252} \mathrm{No}\left(T_{1 / 2}=2.4 \mathrm{~s}\right)$, which was produced under similar conditions using a ${ }^{206} \mathrm{~Pb}$ target. Successful spectroscopy was achieved, with implantation rates of less than one atom per second delivered to the buffer-gas stopping cell indicating the high efficiency of our set-up (Methods). We observed a signal displacement - the isotope shift-of about $0.32 \mathrm{~cm}^{-1}$, which is due to a difference in nuclear size and shape (S.R. et al., manuscript in preparation).

To further confirm the identification of the ${ }^{1} \mathrm{P}_{1}$ state, we measured the saturation characteristics of the first-step resonance; see Fig. 2. Saturation was observed at rather low photon fluxes, which is a clear indication of a sizable dipole-transition amplitude. We obtained a corresponding Einstein coefficient for spontaneous emission of $A=4.2_{-2.8}^{+2.6} \times 10^{8} \mathrm{~s}^{-1}$ by fitting a rate-equation model (P.C. et al., manuscript in preparation) to the saturation data. This value is in agreement with various theoretical predictions (Table 1), which supports our claim that the short-lived $5 f^{4} 7 s 7 p^{1} \mathrm{P}_{1}$ atomic state was observed.

We also observed several high-lying Rydberg states in ${ }^{254} \mathrm{No}$ (Fig. 3a). The first excitation step was set to the resonance at $\bar{\nu}_{1}=29,961.457 \mathrm{~cm}^{-1}$, whereas the second step was scanned with a dye laser in the range $23,460-23,503 \mathrm{~cm}^{-1}$. Two pronounced peaks, potentially members of

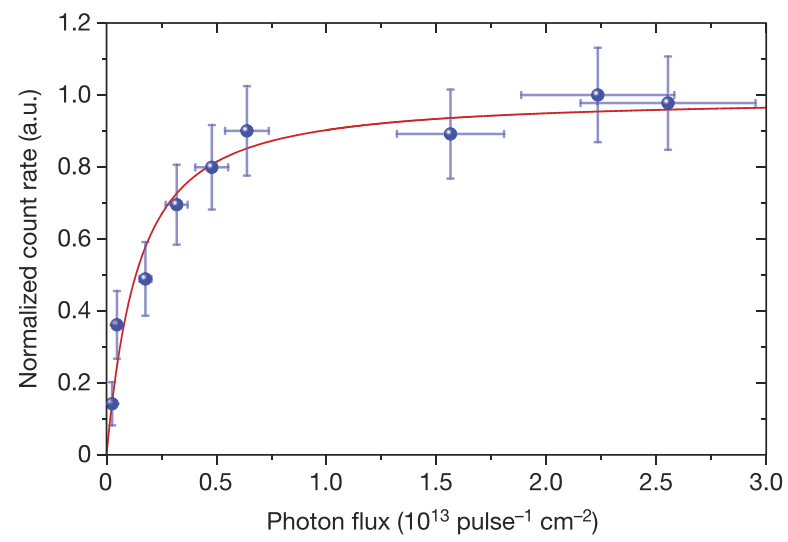

Figure 2 Saturation characteristics of the first-step resonance for ${ }^{254}$ No. $\alpha$-Decay count rates at the resonance peak, normalized to the maximum value, are displayed against the photon flux of the first excitation step. Laser bandwidth, $0.18 \mathrm{~cm}^{-1}$; a.u., arbitrary units. The flux for the second step was kept at $7.3 \times 10^{15}$ photons per pulse per square centimetre during this measurement. A best-fit to the data according to a rate-equation model is also shown (solid line). All error bars indicate \pm 1 s.d. 


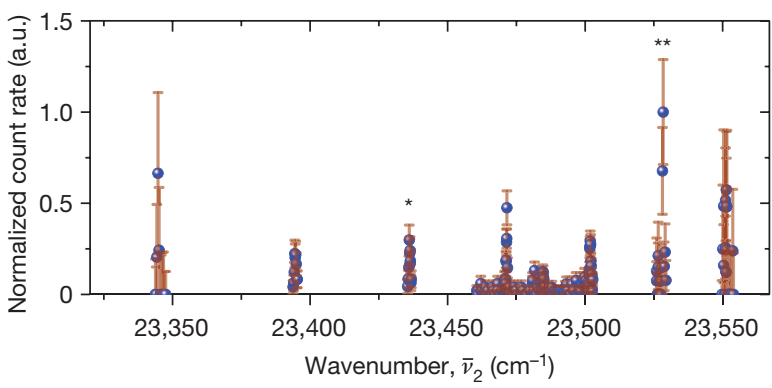

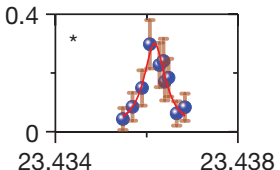

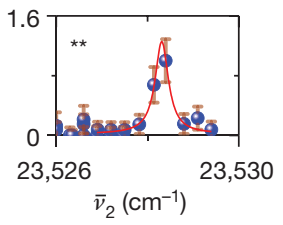

b

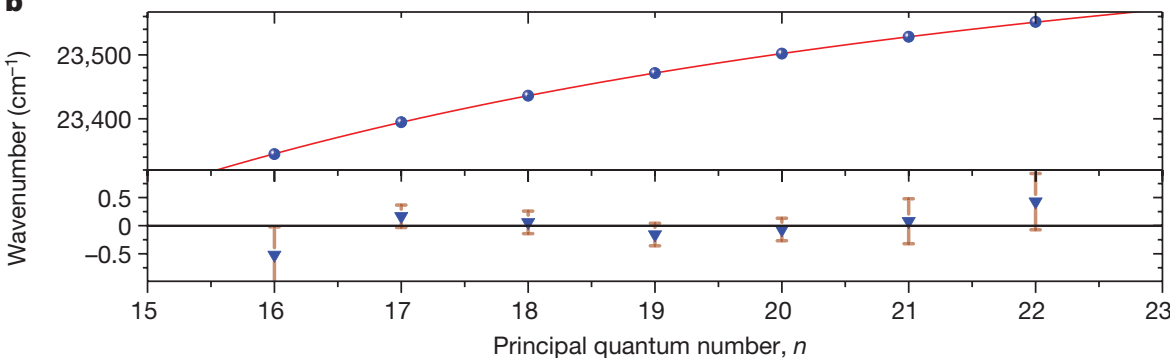

Figure 3 | Observed high-lying Rydberg states in nobelium. a, ${ }^{254} \mathrm{No}$ $\alpha$-decay count rates, normalized to the maximum value, versus the excitation-energy equivalent wavenumber for the second-step excitation, $\bar{\nu}_{2}$. First-step excitation, $\bar{\nu}_{1}=29,961.457 \mathrm{~cm}^{-1}$. Two selected Rydberg resonances with Lorentzian-profile fits (solid lines) to the data are shown on the right, indicated by corresponding asterisks in the left panel. b. Top, the position of seven high-lying Rydberg states (spheres) as a function of the principal quantum number $n$ and a corresponding best fit (solid line) according to the Rydberg-Ritz formula. Bottom, residuals of the fit (triangles). All error bars indicate \pm 1 s.d. the same Rydberg series, were observed. Sampling in an extended scan range revealed further peaks belonging to the same series, which could not be assigned unambiguously.

In the absence of perturbations originating from the interaction with buffer-gas atoms, the transition energies from the excited ${ }^{1} \mathrm{P}_{1}$ state to Rydberg states $h \nu_{2}(n)$ follow a trend described by the Rydberg formula ${ }^{18}: h \nu_{2}(n)=h \nu_{\text {lim }}-R_{m} /(n-\delta)^{2}$. Here, $h \nu_{\text {lim }}=E_{\mathrm{IP}}-h \nu_{1}$ denotes the ionization limit for the excited state, $E_{\mathrm{IP}}$ is the ionization potential, $h$ is the Planck constant, $\nu_{1,2}=c \bar{\nu}_{1,2}=c / \lambda_{1,2}$ are transition frequencies, with $c$ denoting the speed of light, $n$ is the principal quantum number of the valence electron, $R_{m}$ is the reduced-mass Rydberg constant for ${ }^{254}$ No and $\delta$ is the quantum defect. In the firstorder Ritz expansion ${ }^{25}$, the quantum defect can be expressed as $\delta(n)=\delta_{0}+B /\left(n-\delta_{0}\right)^{2}$ with the fitting parameters $\delta_{0}$ and $B$. In the upper panel of Fig. $3 \mathrm{~b}$, the positions of the observed peaks are plotted against their principal quantum number $n$. The assignment of $n$ was based on restricting the quantum defect to be between 0 and 1 (that is, arbitrary), because the outcome of the analysis remained unaffected by this choice. The best fit to the data showed a convergence towards the value $\bar{\nu}_{\lim }=\nu_{\lim } / c=23,785_{-1}^{+11} \mathrm{~cm}^{-1}$. From higher-order corrections in the quantum defect, we estimated a maximum systematic error of $+10 \mathrm{~cm}^{-1}$ for the given value. Shifts in the atomic spectra due to the buffer-gas pressure were neglected because these are expected to be not greater than $0.5 \mathrm{~cm}^{-1}$. However, experimental observations when delaying the non-resonant ionizing laser pulse suggest a fast quenching of the ${ }^{1} \mathrm{P}_{1}$ state, induced by buffer-gas collisions, into a potentially longlived, but energetically very close, atomic state. Hence, the observed series is not necessarily excited from the ${ }^{1} \mathrm{P}_{1}$ state. Possible candidates are ${ }^{3} \mathrm{D}_{3}$ or even a ${ }^{3} \mathrm{D}_{2}$ state, located $159 \mathrm{~cm}^{-1}$ and $1,278 \mathrm{~cm}^{-1}$ below the ${ }^{1} \mathrm{P}_{1}$ state according to ref. 6 , respectively. We therefore include a lower limit for the ionization potential of nobelium as $\bar{\nu}_{\text {lim }}+\bar{\nu}_{1}-1,278 \mathrm{~cm}^{-1}=52,467 \mathrm{~cm}^{-1}$, corresponding to $6.505 \mathrm{eV}$. The upper limit is $\bar{\nu}_{\text {lim }}+\bar{\nu}_{1}=53,757.5 \mathrm{~cm}^{-1}(6.665 \mathrm{eV})$. This upper limit derives from the fact that any populated state energetically lying above the ${ }^{1} \mathrm{P}_{1}$ state would immediately depopulate to the then lower-lying ${ }^{1} \mathrm{P}_{1}$ state and cannot lead (in this context) to delayed resonance ionization. Various theoretical predictions are compared with our experimental value for the ionization potential in Table 1. Our experimental value continues the trend of increasing ionization potential along the heaviest actinides $^{1,26}$ and is substantially higher than the value recently reported for the heavier element lawrencium ${ }^{15}$. In analogy to the lanthanides, the lighter homologues, this corroborates the scenario of closed $5 f$ and $7 s$ atomic shells in nobelium.

In summary, we successfully performed laser spectroscopy of the element nobelium using the ultra-sensitive and highly efficient RADRIS technique. This provides a basis for future experiments, in which atomic-level energies of nobelium, including the first ionization potential, can be determined with unprecedented precision. The data provide the foundation for future theoretical work, which in turn guides experiments. This work opens up the possibility for laser spectroscopy of the heaviest elements, including those beyond nobelium that are accessible in even lower yield and have a different atomic structure. An example is the heaviest actinide element, lawrencium, which is now within reach of such spectroscopy studies. Moreover, isotope shift and hyperfine structure measurements ${ }^{27}$ of nobelium isotopes are now feasible, and would constitute a valuable contribution to nuclear structure studies of deformed nuclei of elements in the region of the periodic table near the super-heavy elements-elements that exist only as a result of nuclear shell structure. These sophisticated alternatives to established in-beam and decay spectroscopy approache ${ }^{28}$ provide complementary information on single-particle and collective properties, as well as spin assignments, and will enable critical testing and revision of state-ofthe-art nuclear models.

Online Content Methods, along with any additional Extended Data display items and Source Data, are available in the online version of the paper; references unique to these sections appear only in the online paper.

Received 16 April; accepted 25 July 2016.

Published online 28 September 2016.

1. NIST ASD Team. Atomic Spectra Database version 5.3, http://physics.nist.gov/ asd (NIST, accessed March 2016).

2. Eliav, E., Fritzsche, S. \& Kaldor, U. Electronic structure theory of the superheavy elements. Nucl. Phys. A 944, 518-550 (2015).

3. Schwerdtfeger, P., Pašteka, L. F., Punnett, A. \& Bowman, P. O. Relativistic and quantum electrodynamic effects in superheavy elements. Nucl. Phys. A 944, 551-577 (2015). 
4. Backe, H., Lauth, W., Block, M. \& Laatiaoui, M. Prospects for laser spectroscopy, ion chemistry and mobility measurements of superheavy elements in buffer-gas traps. Nucl. Phys. A 944, 492-517 (2015).

5. Indelicato, P., Santos, J. P., Boucard, S. \& Desclaux, J.-P. QED and relativistic corrections in superheavy elements. Eur. Phys. J. D 45, 155-170 (2007).

6. Borschevsky, A. et al. Predicted spectrum of atomic nobelium. Phys. Rev. A 75, 042514 (2007)

7. Liu, Y., Hutton, R. \& Zou, Y. Atomic structure of the super-heavy element No I $(Z=102)$. Phys. Rev. A 76, 062503 (2007).

8. Dzuba, V. A., Safronova, M. S. \& Safronova, U. I. Atomic properties of superheavy elements No, Lr, and Rf. Phys. Rev. A 90, 012504 (2014)

9. Worden, E. F., Blaise, J., Fred, M., Trautmann, N. \& Wyart, J.-F. in The Chemistry of the Actinide and Trans-actinide Elements Vol. 3 (eds Morss, L. R. et al.) Ch. 16.4 (Springer, 2008)

10. Sewtz, M. et al. First observation of atomic levels for the element fermium $(Z=100)$. Phys. Rev. Lett. 90, 163002 (2003).

11. Sewtz, M. et al. Resonance ionization spectroscopy of fermium $(Z=100)$. Spectrochim. Acta B 58, 1077-1082 (2003).

12. Backe, $H$. et al. Laser spectroscopic investigation of the element fermium ( $Z=100)$. Hyperfine Interact. 162, 3-14 (2005).

13. Nagame, Y., Kratz, J. V. \& Schädel, M. Chemical studies of elements with $Z \geq 104$ in liquid phase. Nucl. Phys. A 944, 614-639 (2015).

14. Türler, A., Eichler, R. \& Yakushev, A. Chemical studies of elements with $Z>104$ in gas phase. Nucl. Phys. A 944, 640-689 (2015).

15. Sato, T. K. et al. Measurement of the first ionization potential of lawrencium, element 103. Nature 520, 209-211 (2015).

16. Lauth, W. et al. Resonance ionization spectroscopy in a buffer gas cell with radioactive decay detection, demonstrated using ${ }^{208}$ TI. Phys. Rev. Lett. 68, 1675-1678 (1992)

17. Backe, H. et al. Towards optical spectroscopy of the element nobelium $(Z=102)$ in a buffer gas cell. Eur. Phys. J. D 45, 99-106 (2007).

18. Letokhov, V. S. Laser Photoionization Spectroscopy (Academic Press, 1987).

19. Backe, H. et al. Isotope shift measurements for superdeformed fission isomeric states. Phys. Rev. Lett. 80, 920-923 (1998).

20. Backe, H. et al. Stability of superdeformation for americium fission isomers as function of the neutron number. Nucl. Phys. A 690, 215-218 (2001)

21. Laatiaoui, M. et al. On laser spectroscopy of the element nobelium $(Z=102)$ Eur. Phys. J. D 68, 71-77 (2014).

22. Hofmann, S. \& Münzenberg, G. Discovery of the heaviest elements. Rev. Mod. Phys. 72, 733-767 (2000).
23. Lautenschläger, F. et al. Developments for resonance ionization laser spectroscopy of the heaviest elements at SHIP. Nucl. Instrum. Methods B $\mathbf{3 8 3}$, 115-122 (2016).

24. Fritzsche, S. On the accuracy of valence-shell computations for heavy and super-heavy elements. Eur. Phys. J. D 33, 15-21 (2005).

25. Martin, W. C. Series formulas for the spectrum of atomic sodium (Na I). J. Opt. Soc. Am. 70, 784-788 (1980)

26. Wendt, K., Gottwald, T., Mattolat, C. \& Raeder, S. Ionization potentials of the lanthanides and actinides - towards atomic spectroscopy of super-heavy elements. Hyperfine Interact. 227, 55-67 (2014).

27. Campbell, P., Moore, I. D. \& Pearson, M. R. Laser spectroscopy for nuclear structure physics. Prog. Part. Nucl. Phys. 86, 127-180 (2016).

28. Herzberg, R.-D. et al. Nuclear isomers in superheavy elements as stepping stones towards the island of stability. Nature 442, 896-899 (2006).

29. Sugar, J. Revised ionization energies of the neutral actinides. J. Chem. Phys. 60, 4103 (1974)

Acknowledgements We thank the staff of the GSI ion source and accelerator for the preparation of a stable ${ }^{48} \mathrm{Ca}$ beam and the staff of the target laboratory for providing high-quality targets. We acknowledge the technical support of J. Maurer, H. G. Burkhard, D. Racano, L. Braisz, D. Reemts, C. Droese, B. Schausten and I. Kostyuk. We thank P. Thirolf for his suggestions and comments. This work was supported by the German Federal Ministry of Education and Research under contracts 06MZ169I, 06LM236I, FAIR NuSTAR 05P09RDFN4, 05P12RDFN8 and 05P15RDFN1; by the GSI; and by the Helmholtz-Institut Mainz.

Author Contributions W.L., H.B., M.B., T.W., P.V.D., C.E.D., M.H. and A.Y. provided experimental equipment. M.L., F.L., P.C., S.R., W.L., P.K., M.B., F.P.H., D.A., C.W., A.K.M., B.C., R.F., F.G., O.K., J.K., J.E., S.G. and E.M.R. performed the experiments. F.L., P.C., H.B., S.R. and M.L. analysed the data. M.L. wrote the manuscript with input from all authors.

Author Information Reprints and permissions information is available at www.nature.com/reprints. The authors declare no competing financial interests. Readers are welcome to comment on the online version of the paper. Correspondence and requests for materials should be addressed to M.L. (M.Laatiaoui@gsi.de).

Reviewer Information Nature thanks V. Fedosseev and L. Visscher for their contribution to the peer review of this work. 


\section{METHODS}

Production of ${ }^{252} \mathrm{No}$ and ${ }^{254} \mathrm{No}$. The experiments described herein were carried out behind the velocity filter SHIP ${ }^{22}$ at the linear accelerator (UNILAC) of GSI Helmholtzzentrum für Schwerionenforschung in Darmstadt. The isotopes ${ }^{252,254} \mathrm{No}$ were produced in the complete-fusion evaporation reactions ${ }^{206} \mathrm{~Pb}\left({ }^{48} \mathrm{Ca}, 2 \mathrm{n}\right){ }^{252} \mathrm{No}$ and ${ }^{208} \mathrm{~Pb}\left({ }^{48} \mathrm{Ca}, 2 \mathrm{n}\right){ }^{254} \mathrm{No}$ at UNILAC beam energies of $217 \mathrm{MeV}$ with cross-sections of $515_{-47}^{+80} \mathrm{nb}$ and $2,050_{-340}^{+460} \mathrm{nb}$ (ref. 30), using lead sulfide (PbS) targets ${ }^{31}$ with average thicknesses of $460 \mu \mathrm{g} \mathrm{cm}^{-2}$ and $470 \mathrm{\mu g} \mathrm{cm}^{-2}$, respectively, which remained constant throughout the measurements. The ${ }^{48} \mathrm{Ca}^{10+}$ beam from the UNILAC exhibited a macro-pulse structure of $5 \mathrm{~ms}$ beam-on and $15 \mathrm{~ms}$ beam-off. Beam currents were typically 0.7 particle microamperes (about $4.4 \times 10^{12}$ particles per second). For laser spectroscopy experiments, the beam was further chopped in accordance with user-defined measurement cycles. In the case of ${ }^{254} \mathrm{No}$, the implantation rate of the fusion products delivered to the experiment was repeatedly checked by a retractable position-sensitive 16-strip silicon detector placed at the focal plane of SHIP.

Radiation detected resonance ionization spectroscopy. The fusion-evaporation residues with a mean kinetic energy of about $41 \mathrm{MeV}$ (ref. 23) were separated from the ${ }^{48} \mathrm{Ca}$ primary beam by the velocity filter SHIP and subsequently thermalized inside the optical cell, a buffer-gas stopping cell filled with 95-mbar argon of ultrahigh purity (99.9999\%). The cell was separated from the vacuum of the SHIP by a 3.5- $\mu \mathrm{m}$-thick entrance foil (Mylar) on a support grid; see Extended Data Fig. 1. The geometrical transparency of the grid amounts to $90.3 \%$. A substantial fraction of the stopped fusion products remained in a positive charged state and was collected during the accumulation time of every measurement cycle onto a catcher filament - a tantalum wire with a diameter of $125 \mu \mathrm{m}$. The filament was heated for $300 \mathrm{~ms}$ to a temperature of about $1,350 \mathrm{~K}$, triggering the evaporation of neutral nobelium atoms for subsequent two-step laser ionization ${ }^{21}$. Ions produced during this process were promptly guided by suitable electric fields to a particle detector-a passivated implanted planar silicon (PIPS) semiconductor detector-with which the characteristic $\alpha$-decay or spontaneous fission of the studied nobelium isotopes and their descendants was registered ${ }^{17,21,23}$. The optimum accumulation time depends on the half-life of the isotope under consideration and was $25 \mathrm{~s}$ (ref. 32) for ${ }^{254} \mathrm{No}$ with $T_{1 / 2}=51.2 \pm 0.4 \mathrm{~s}$ (ref. 28). The two-step laser ionization took place during a 5-s time window every cycle, while the primary ion beam was switched off. Correspondingly, 3-s beam-on and 3-s beam-off periods were chosen for the shortlived isotope ${ }^{33}{ }^{252} \mathrm{No}\left(T_{1 / 2}=2.42 \pm 0.06 \mathrm{~s}\right)$. We further introduced 'waiting' cycles by interrupting the data acquisition while changing the laser frequencies. In Fig. 1a, for instance, a 'waiting' cycle of $5 \mathrm{~min}$ was chosen, which was necessary especially in the case of ${ }^{254} \mathrm{No}$, to minimize residual ${ }^{254} \mathrm{No} \alpha$-decay events that might lead to counts not belonging to the chosen laser frequency. The absolute temperature of the filament was monitored using a fast infrared pyrometer (LumaSense, IMPAC IS 6 Advanced). The temperature was adjusted to the nominal value when necessary to prevent overheating, which was observed to lead to increased background in preparatory experiments.

Laser set-up for nobelium spectroscopy. The extended level search in nobelium was carried out using four tunable excimer laser-pumped dye lasers (Lambda Physik, FL and LPD series, bandwidth of $5.5 \pm 0.5 \mathrm{GHz}$ ) and an optical parametric oscillator (OPO) system (GWU-Lasertechnik, VisIR2, bandwidth of about $90 \mathrm{GHz}$ ) pumped by a frequency-tripled Nd:YAG laser (Continuum, Powerlite DLS 8050). Except for the 50-Hz Nd:YAG laser, all the other pump lasers were operated at a repetition rate of $100 \mathrm{~Hz}$. The duration of the laser pulse was at most $18 \mathrm{~ns}$ (FWHM) with a jitter of $<11 \mathrm{~ns}$ for all pulses. The dye lasers were set up to scan in the range $25,000-31,000 \mathrm{~cm}^{-1}$. However, during the level search in nobelium, the scans were conducted mainly in the ultraviolet region of the optical spectrum. With a mean step size of about $0.89 \mathrm{~cm}^{-1}$, we thereby covered more than twice the spectral range from $28,887 \mathrm{~cm}^{-1}$ to $30,530 \mathrm{~cm}^{-1}$. With the OPO system, operated in a frequency-mixing mode, and by choosing a scan step size of $3 \mathrm{~cm}^{-1}$, adapted to the laser bandwidth, multiple scans from $30,000 \mathrm{~cm}^{-1}$ to $33,191 \mathrm{~cm}^{-1}$ were conducted. The laser wavelengths were continuously monitored with a wavelength meter (HighFinesse-Ångstrom, WS/7-UVU) that was calibrated to an internal neon lamp. Laser pulse energies in excess of $0.1 \mathrm{~mJ}$ were repeatedly measured at the optical cell for all tunable lasers. For efficient use of beam time, the lasers were operated simultaneously in different wavelength ranges. They were synchronized with excimer laser synchronization units (Lambda Physik, LPA 97) with respect to the ionizing laser-an excimer laser (Lambda Physik, LPX220) delivering an average pulse energy of $45 \mathrm{~mJ}$ of broadband laser light in the wavelength range $349-353 \mathrm{~nm}$ at the optical cell. The total photon energy available for ionization was much higher than all theoretical predictions and extrapolations of the ionization potential of nobelium (Table 1). The scans near the ionization potential were performed by replacing the excimer ionizing laser with a tunable dye laser scanning in the blue range of the optical spectrum. Only two dye lasers were operated simultaneously. In addition, the dye lasers enabled the use of intra-cavity etalons and thus a narrowing of the laser bandwidth down to $1.2 \mathrm{GHz}$. The narrow resonance shown in Fig. 1a (spheres) was recorded using this option. More details on the laser systems used can be found in ref. 23.

RADRIS efficiency. The overall efficiency of the set-up is defined as the ratio of the nobelium decay count rate measured with the PIPS detector $A_{\text {RIS }}$ at the maximum of a resonance to the implantation rate of nobelium ions delivered to the optical cell $A_{\text {ion }}$, both normalized to the intensity of the primary beam. The spatial distribution of the ${ }^{254} \mathrm{No}$ ions delivered to the experiment is best described by a two-dimensional Gaussian distribution with $\sigma_{x}=22 \mathrm{~mm}$ and $\sigma_{y}=5.7 \mathrm{~mm}$ on the basis of a measurement with the position-sensitive 16-strip silicon detector exhibiting an active area of $80 \mathrm{~mm} \times 35 \mathrm{~mm}$. The ion implantation rate was extracted from the $\alpha$-decay count rate $A_{\alpha}$ measured with this detector according to $A_{\text {ion }}=$ $A_{\alpha} /\left(\varepsilon_{\alpha} \varepsilon_{\Omega} \varepsilon_{\text {daq }}\right)$, with $\varepsilon_{\alpha}=0.9$ the $\alpha$-decay probability for ${ }^{254} \mathrm{No}, \varepsilon_{\Omega}=0.55$ the solid angle coverage for $\alpha$ decays from implanted ${ }^{254}$ No recoils ${ }^{23}$, and $\varepsilon_{\text {daq }}=0.77$ the efficiency for data acquisition, which was limited to the beam-off time windows during which data recording occurred. On average, decay rates in the PIPS detector of $0.39 \pm 0.05$ and $0.048 \pm 0.006$ per second per particle microampere $\left(6.2 \times 10^{12}{ }^{48} \mathrm{Ca}\right.$ projectiles per second $)$ were obtained for ${ }^{254} \mathrm{No}$ and ${ }^{252} \mathrm{No}$, respectively. With these numbers, an overall efficiency of the apparatus of $6.4 \% \pm 1 \%$ and $3.3 \% \pm 1 \%$ was calculated for ${ }^{254} \mathrm{No}$ and ${ }^{252} \mathrm{No}$, respectively, demonstrating the high efficiency of the applied spectroscopy technique. The difference in the quoted numbers is mainly due to the half-lives of the isotopes under investigation. In the spectroscopy of ${ }^{252} \mathrm{No}$, a shortest possible beam-off period of $3 \mathrm{~s}$ was applied. Even though the measurement cycle was optimized to minimize the impact of the half-life on the overall efficiency, the spectroscopy of ${ }^{252}$ No turned out to be less efficient than that of ${ }^{254} \mathrm{No}$.

30. Oganessian, Yu. Ts, et al. Measurements of cross sections for the fusionevaporation reactions ${ }^{204,206,207,208} \mathrm{~Pb}+{ }^{48} \mathrm{Ca}$ and ${ }^{207} \mathrm{~Pb}+{ }^{34} \mathrm{~S}$ : decay properties of the even-even nuclides ${ }^{238} \mathrm{Cf}$ and ${ }^{250} \mathrm{No}$. Phys. Rev. C 64, 054606 (2001).

31. Kindler, B. et al. Chemical compound targets for SHIP on heated carbon backings. Nucl. Instrum. Methods A 561, 107-111 (2006).

32. Laatiaoui, M. et al. Perspectives for laser spectroscopy of the element nobelium. Hyperfine Interact. 227, 69-75 (2014).

33. Sulignano, B. et al. Identification of a $\mathrm{K}$ isomer in ${ }^{252} \mathrm{No}$. Eur. Phys. J. A 33 , 327-331 (2007). 


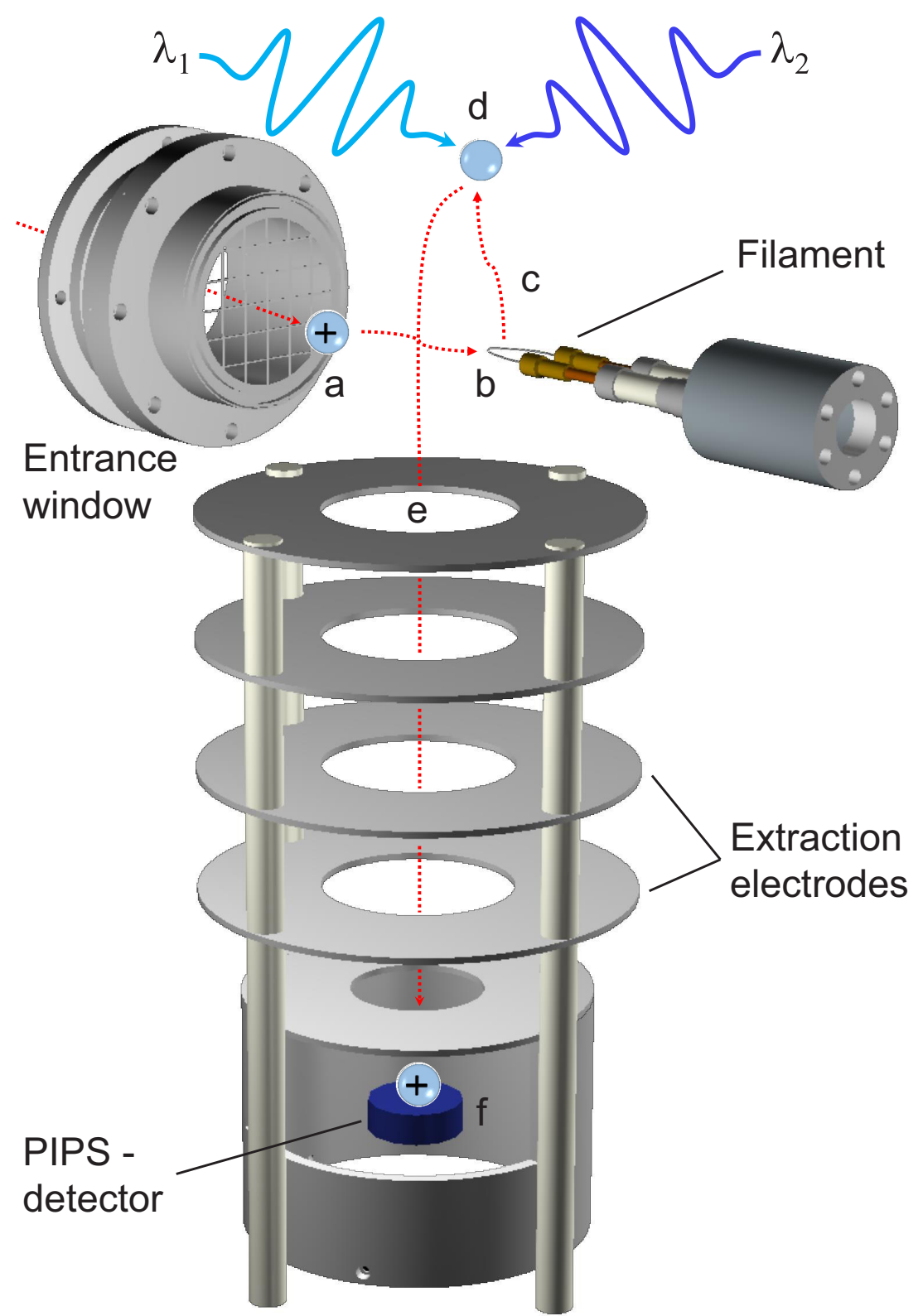

Extended Data Figure 1 | Principle of the RADRIS technique. Laser spectroscopy on radionuclides (spheres) after their production and transmission through the velocity filter SHIP ${ }^{22}$. a, Thermalization of the fusion products in the buffer gas; $\mathbf{b}$, accumulation on the catcher filament; c, re-evaporation from the filament; $\mathbf{d}$, two-step photoionization of neutral atoms; $\mathbf{e}$, accumulation of re-ionized fusion products on the PIPS detector; f, decay detection. 
Extended Data Table 1 | Uncertainties on the value of the ${ }^{254}$ No first-step resonance

Origin Uncertainty $\left(\mathrm{cm}^{-1}\right) \times 10^{-3}$

Fit

$\pm 4.2$

Wav elength measurements

$\pm 5.0$

Pressure shift

$+34.2$

The peak-position in the narrow-band-scan data are extracted from a best fit based on a rate-equation model. The value for the wavelength measurement represents the accuracy of the wavelength meter in multimode-fibre operation. A conservative value for the pressure shift is taken from ref. 16 . 